\title{
Evaluación Ergonómica en el Área Termoencogible de una Empresa Fabricante De Pintura Venezolana
}

\author{
ERGONOMIC EVALUATION IN THE HEAT SHRINKS AREA OF A VENEZUELAN PAINT MANUFACTURER
}

Víctor Castillo-Guerrero', Norys Suárez², Evelin Escalona ${ }^{3}$

1. Doctorando en Prevención de Riesgos Laborales. Departamento de Sociología de la Universidad Pública de Navarra (España). Profesor-Investigador adscrito al Programa de Ingeniería, Arquitectura y Tecnología. Vicerrectorado de Planificación y Desarrollo Social. Universidad Nacional Experimental de los Llanos Occidentales Ezequiel Zamora (UNELLEZ-VPDS), Barinas. Venezuela. e-mail: castillovictordaniel@hotmail.com

2. Médica Especialista en Salud Ocupacional e Higiene del Ambiente Laboral. Investigadora independiente. Egresada postgrado de la Facultad de Ciencias de la Salud de la Universidad de Carabobo.e-mail: norys1807@hotmail.com

3. Centro de Estudios en Salud de los Trabajadores / Universidad de Carabobo. Escuela de Medicina, Facultad de Ciencias de la Salud (Sede Aragua). Maracay-Aragua. Venezuela. e-mail: evelinescalona@gmail.com

\section{RESUMEN}

El objetivo de la investigación fue realizar una evaluación ergonómica a los puestos de trabajo del área de Termoencogible, identificando los factores peligrosos y los determinantes de lesiones musculoesqueléticas en una empresa fabricante de pintura ubicada en el sur del estado Aragua, Venezuela. Métodos: Fue utilizada la triangulación de investigación (cualitativa-cuantitativa); Métodos Ergonómicos (Deparis; REBA; NIOSH) con Observación de Puestos, entrevista colectiva; Grupos de Exposición Homogéneos y Análisis Antropométrico. La población laboral fue de 200 trabajadores. Se seleccionó de forma intencional el área Termoencogible, donde trabajan 06 hombres que fueron los sujetos de investigación. Resultados: el diagnóstico DEPARIS muestra inconformidad y peligro en el ritmo de trabajo y los aspectos organizacionales. El esfuerzo físico y la carga mental representan grandes exigencias para los trabajadores. Las puntuaciones REBA obtenidas revelan riesgo en dos de los tres puestos de trabajo, el ritmo de trabajo exige rotaciones del tronco de tres a seis veces por minutos, movimientos repetitivos, y levantamiento de cargas que supera en cuatro veces la determinada por la Ecuación de NIOSH. Finalmente, los determinantes presentes en el puesto de trabajo que podrían explicar los trastornos musculoesqueléticos (TME) son los problemas de organización del trabajo, referidos a la supervisión estricta, carga de trabajo excesiva, déficit de personal. En cuanto a los medios y equipos de trabajo, el diseño del puesto es inadecuado por lo que se requiere la automatización y mecanización de los procesos y de esa manera evitar la manipulación de cargas fuera de la zona neutral del cuerpo.

(Castillo-Guerrero V, Suárez N, Escalona E, 2013. Evaluación Ergonómica en el Área Termoencogible de una Empresa Fabricante de Pintura Venezolana. Cienc Trab. May-Ago; 15 [47]: 57-62).

Palabras claves: CONDICIONES DE TRABAJO; TRABAJO REPETITIVO; INGENIERÍA HUMANA.

\section{ABSTRACT}

The objective of the research was to conduct an ergonomic assessment to jobs of heat shrink area, identifying hazardous factors and determinants of musculoskeletal injuries at a paint manufacturing company located in the south of Aragua state, Venezuela. Methods: It was used the triangulation of research (qualitative and quantitative) Ergonomics Methods (Deparis, REBA, NIOSH) with observation of positions, collective interviews, Homogeneous Exposure Groups and Anthropometric Analysis. The workforce was 200 employees. They were selected intentionally from the heat shrink area where 06 men work, who were research subjects. Results: DEPARIS diagnosis shows discontent and danger in the pace of work and organizational aspects. The physical and mental workloads are major requirements for workers. REBA scores obtained reveals risk in two of the three jobs, work rhythm requires trunk rotations three to six times per minute, repetitive motion, and lifting of loads that exceeds four times the equation determined by the NIOSH. Finally, the determinants present in the workplace that could explain musculoskeletal disorders (MSDs) are the problems of work organization, referring to strict supervision, excessive workload, staff shortages. As for the media and work teams, job design is inadequate so it is required automation and mechanization of processes and thereby avoiding handling loads out of the neutral zone of the body.

Key words: WORKING CONDITIONS, REPETITIVE WORK, HUMAN ENGINEERING
Correspondencia / Correspondence:

Victor Castillo Guerrero

Avda. 23 de Enero. Redoma Punto Fresco,

Barinas. Venezuela

Tel.: +58 2735336227

e-mail: castillovictordaniel@hotmail.com

Recibido: 08 de Abril de 2013 / Aceptado: 20 de Mayo de 2013

\section{INTRODUCCIÓN}

La forma de trabajar ha cambiado en función de los avances científicos y según las necesidades de la sociedad. El trabajo es un hecho social que transforma a los individuos, tiene implícitos riesgos para la salud ya que las deficiencias en las condiciones de trabajo pueden generar fatiga y lesiones osteomusculares. ${ }^{1,2} \mathrm{La}$ salud de los trabajadores y la seguridad en los centros de trabajo debe estar garantizada a fin de evitar los accidentes y las enfer- 
medades ocupacionales; por ello en Venezuela, según Yanes ${ }^{3}$, se han dado cambios en la regulación legal reciente ocasionando un creciente interés en el empleador para mejorar las condiciones de salud y seguridad en el trabajo a fin de evitar sanciones del Instituto Nacional de Prevención Salud y Seguridad Laborales (INPSASEL).

Escalona, Yonusg, González y colaboradores ${ }^{4}$, refieren que las condiciones de trabajo seguras implican la aplicación de la ergonomía adaptando el trabajo a las personas y evitando así los problemas físicos, mentales y emocionales. En este contexto, una empresa ubicada en Santa Cruz de Aragua (Venezuela), destinada a la fabricación y comercialización de pinturas de uso doméstico e industrial, consideró necesario abordar una situación problemática que surge como consecuencia de quejas reiteradas de los trabajadores, quienes plantean la existencia de condiciones de trabajo peligrosas.

La empresa objeto de estudio tiene 200 trabajadores distribuidos en siete departamentos; ellos son: a) Administración; b) Laboratorio; c) Materia Prima; d) Taller; e) Almacén; f) Mantenimiento y g) Producción. El Departamento de Producción está conformado por ocho áreas de trabajo, donde labora 35\% del personal (70 personas). Sin embargo, pareciera que una de las áreas de trabajo resulta desagradable y peligrosa para todos los trabajadores que evitan ser trasladados a esa zona. Es el caso del área de Termoencogible, donde se realiza el empaquetamiento de los envases llenos de pintura que luego son ubicados en el área de almacenamiento.

El área tiene tres puestos de trabajo; es un lugar que pareciera representar para los trabajadores exigencias físicas y ergonómicas. Los trabajadores se quejan por dolores de espalda, de los hombros, la cintura y de los pies. Ello, a su vez, ocasiona consultas médicas reiteradas y/o reposos médicos. Una observación preliminar realizada desde el Servicio Médico de la empresa en el área de Termoencogible encontró que los trabajadores realizan actividades que Messing, Vogel, Parra, Grönkvis y Lagerlöf ${ }^{5}$ vinculan con los trastornos musculoesqueléticos, esto es: a) movimientos repetitivos; b) flexión y rotación del cuello, tronco y miembros superiores e inferiores y; c) levantamiento de carga.

Durante el 2008, en el servicio médico de la empresa se realizaron 782 consultas, donde 230 (30\%) fueron por lesiones musculoesqueléticas, 142 por afecciones respiratorias (18\%), el dolor de cabeza y las afecciones gastrointestinales representan 17\%. Las lesiones musculoesqueléticas en su mayoría proceden del personal obrero, así como las afecciones respiratorias y gastrointestinales y la cefalea predomina en el personal administrativo. La región del cuerpo más afectada de trastornos musculoesqueléticas (TME), según los registros médicos de la empresa, fue la espalda alta y baja (región dorso-lumbar) que representa 78\%, las otras zonas de molestias representan 22\% (hombros, cuello, rodilla, pies, piernas y manos).

La situación antes descrita permitió inferir sobre la existencia de deficiencias ergonómicas en el área Termoencogible y se planteó las siguientes interrogantes: ¿Qué factores de riesgo están presentes en el área Termoencogible que puedan generar trastornos musculoesqueléticos? ¿Cumple el área de trabajo en estudio con las condiciones ergonómicas básicas? ¿Qué mejoras pueden incorporarse en el área de trabajo en estudio a fin de garantizar la salud y la seguridad en el trabajo? Por lo antes expuesto, se traza como objetivo realizar una evaluación ergonómica a los puestos de trabajo del área de Termoencogible, con el propósito de identificar los factores peligrosos y los determinantes de lesiones musculoesqueléticas en el área Termoencogible en una empresa fabricante de pintura.

\section{METODOLOGÍA}

Se diseñó un estudio de intervención y se utilizó el enfoque de la ergonomía centrada en el análisis del trabajo de Escalona y colaboradores (obra citada). La Evaluación Ergonómica fue realizada desde un enfoque multimétodo (cuantitativo-cualitativo). La muestra es intencional, se tomó el área Termoencogible debido a la preocupación de la Gerencia de Seguridad y Recursos Humanos de la empresa, unido a las recurrentes quejas de los trabajadores y a las consultas vinculadas con molestias musculoesqueléticas. Dicha área contiene una máquina donde laboran un operador, cuatro ayudantes y un supervisor. Ellos pueden ocupar cualquiera de los tres puestos de trabajo indistintamente. Se realizó un estudio integral de los puestos de trabajo incorporando a los trabajadores y usándose una combinación de técnicas (entrevista, observación).

Respecto de las técnicas y métodos ergonómicos resaltan: a) La entrevista individual y la entrevista colectiva con el Método Deparis propuesto por Malchaire ${ }^{6}$; b) la observación de tarea $\mathrm{y}$ determinación del ciclo de trabajo; c) la observación de posturas con el Método de $\mathrm{REBA}^{7}$; d) la evaluación del levantamiento de peso mediante la Ecuación de NIOSH; e) el análisis físico y dimensional de los puestos de trabajo mediante la consideración de la antropometría del trabajador y considerando el límite de alcance; f) determinación de las medidas del puesto de trabajo en relación a los usuarios y finalmente se aplicó el cociente de confort en la posición de trabajo propuesto por Castillo y Escalona. ${ }^{8}$

Las observaciones de los puestos de trabajo se realizaron durante un día típico de trabajo (ocho horas de trabajo) y a máximo nivel de producción, aplicando análisis de la antropometría de forma integral considerando la rotación de todos los trabajadores en los tres puestos de trabajo. El nivel de confort se mide tomando la altura de desplazamiento de la tarea realizada y su relación con las dimensiones corporales (hombros, codos, y manos). La evaluación de las posturas fue con el método REBA para evaluar los riesgos de padecer lesiones asociadas a una postura principalmente de tipo musculoesqueléticas y definiendo la urgencia para las acciones correctivas. ${ }^{7}$

\section{Aspectos éticos}

Se procedió a solicitar el consentimiento informado de los trabajadores para utilizar la información que ellos proporcionaban garantizando la confidencialidad. Los trabajadores firmaron una carta donde aceptaban ser informantes y sujetos de investigación. El trabajo se realizó desde el Servicio Médico de la empresa a petición de la Gerencia General.

\section{RESULTADOS}

El área de trabajo objeto de estudio pertenece a Producción, cuenta con suficiente espacio físico $\left(80 \mathrm{~m}^{2}\right)$, en condiciones limpia, con ventilación e iluminación; tiene tres puestos de trabajo: el primero ocupado por 1 o 2 trabajadores que realizan la tarea de desarme de la paleta, cada paleta contienen 6 camadas ubicadas a diferente altura, siendo la camada 6 la de mayor altura 
$(1,27 \mathrm{~m})$. El puesto 2 está ocupado por un operador que toma los cuatro galones, los coloca en la zona de plastificado para empaquetarlos y los traslada al puesto 3 con ayuda de una cinta trasportadora. El tercer puesto es ocupado por 1 o 2 trabajadores; ellos toman los paquetes de la barra transportadora, los coloca en forma ordenada sobre la paleta comenzando por la camada inferior (0,27 $\mathrm{m}$ del piso). El número de trabajadores del puesto 1 y 3 depende de la producción del día.

\section{Condiciones peligrosas y percepción de los trabajadores}

La edad de los trabajadores osciló entre 21 y 39 años, con excepción del supervisor que tiene 53 años. Todos pertenecen al sexo masculino, la estatura se encuentra entre 1,71 y 1,84 m, un peso entre 69 y $110 \mathrm{Kg}$; la antigüedad en el puesto de trabajo está entre 5 meses y 2 años. Cuatro (04) de los cinco (05) trabajadores, sin contar al supervisor, refieren que el área de Termoencogible es el puesto del área de producción en que ningún trabajador quiere laborar. Ellos indican que se sienten "reventados" y refieren diversos dolores en la espalda, hombro y otras partes de cuerpo.

Las principales zonas de molestias reportadas por los trabajadores al final de la jornada son: en primer lugar, la espalda baja o región lumbar; en segundo lugar, los hombros; en tercer lugar, la espalda alta (región dorsal); en cuarto lugar, la región cervical (cuello); en quinto lugar, los brazos; en sexto lugar, las piernas y, en último lugar, las rodillas. La organización de trabajo es considerada por los trabajadores en situación de alerta; el trabajo se realiza en contrarreloj. Ellos dicen "no nos dejan ni tomar agua porque hay que cumplir con la producción”. La adecuación del trabajo es una situación considerada altamente peligrosa, se exige una intervención inmediata, el trabajo se percibe inadecuado. Ellos dicen "tenemos que agacharnos, se trabaja todo el día parado y eso cansa”. (Ver Cuadro № 1). Los accidentes de trabajo están en alerta, preocupa a los trabajadores el peligro de apisonamiento de los dedos de las manos, cables sueltos y el polvo junto a otros químicos de las áreas cercanas añaden peligro (Ver Cuadro $\mathrm{N}^{\circ} 1$ ). Los comandos y señales no representan peligro alguno (color verde); respecto de las herramientas y material de trabajo, no cuentan con ellas, se dificulta el agarre de envases; situación de alerta debe mejorarse (color amarillo). En relación al trabajo repetitivo, la manipulación de carga y la carga mental se consideran una situación peligrosa, ellos dicen: "el trabajo debe hacerse rápido... la posición es incómoda y nos hace doler los pies". Ellos dicen que el tercero es el "más fuerte". El trabajo es identificado como "aburrido y cansón", que implicaría un alto nivel de exigencia física y esfuerzo (ver Cuadro $\mathrm{N}^{\circ}$ 1). Los factores ambientales (iluminación, ruido, ambiente térmico, vibraciones) no representan peligro alguno y ellos consideran que están conforme (color verde); sin embargo, refieren que se enfrentan a factores psicosociales. Las relaciones de trabajo, el ambiente social, el contenido de trabajo y el ambiente psicosocial que se percibe, son altamente peligrosos (rojo). Ellos dicen: "el supervisor persigue a los trabajadores y no deja ni hablar por un ratito". Agregan "no podemos ir ni al baño porque no hay tiempo para nada”. El área de Termoencogible es considerada en la empresa como la peor área para trabajar. Sostienen que: "nadie quiere estar aquí... uno parece un burrito, no hay oportunidades para crecer ni para nada... por algo será que nadie quiere que lo manden para el termo... uno viene para acá y luego lo que sale es reventado". (Ver Cuadro No 1).
Cuadro 1.

Síntesis de la situación de trabajo en el área de Termoencogible.

Situación de trabajo
1. Las áreas de trabajo
2. La organización técnica entre puestos de trabajo
3. La adecuación del trabajo
4. Los accidentes
5. Los comandos y señales
6. Las herramientas y el material de trabajo
7. El trabajo repetitivo
8. La manipulación de cargas
9. La carga mental
10. La iluminación
11. El ruido
12. El ambiente térmico
13. Riesgos químicos y biológicos
14. Las vibraciones
15. Las relaciones de trabajo entre operarios
16. El ambiente social local y general
17. El contenido del trabajo
18. El ambiente psicosocial

Fuente: trabajadores del área en estudio (06).

Descripción de la actividad de trabajo, ciclo y microciclos Se realiza en tres puestos de forma simultánea. El Puesto 1: el trabajador inicia el ciclo tomando un cartón (soporte), arma un paquete de 4 galones en la barra transportadora tomando los galones de dos en dos hasta descargar la totalidad de la paleta (252 galones). El trabajador del Puesto 2: recibe los envases de la barra transportadora y sujeta el cartón para deslizar los cuatro galones ubicándolos sobre la máquina plastificadora, hala el plástico que se encuentra a 1,8 $\mathrm{m}$ del piso y cubre con ello los cuatro envases, con sus pies activa el descenso de la máquina selladora resultando un paquete que es tomado en el tercer puesto. El trabajador del Puesto 3: recibe y toma estos paquetes con sus dos manos y los va colocando en forma ordenada en una nueva paleta.

El ciclo es descargar, empaquetar y armar una paleta (252 galones); se repite 20 veces al día (9 horas) en casos de mediana producción y 46 veces al día en máximo de producción; varía de 10 a 20 minutos e implica la realización de 63 microciclos. El promedio para descargar y armar una paleta observado en un día de trabajo fue de 15 minutos. Las interrupciones que ocurren en los ciclos son debidas a que se termina el rollo de plastificado, se acumulan los envases en el operador 2 o se le acumulan los paquetes al trabajador 3. Respecto de los Microciclos: El trabajador del Puesto 1 toma un cartón, lo coloca sobre la cinta transportadora y, sobre éste, coloca 4 galones; tiene una duración de 10 a 14 segundos. Puesto 2, el trabajador recibe los 4 envases, los desliza para ubicarlos sobre la máquina plastificadora, hala el plástico que se encuentra a 1,80 metros del piso para cubrir los 4 galones y presionar el botón para activar el descenso de la máquina selladora, para ello, tarda 14 segundos. Por último, el microciclo del Puesto 3 consiste en recibir el paquete de la cinta mecánica procedente del puesto 2 y colocarlo sobre la paleta con una duración promedio de 14 segundos.

\section{Posturas observadas, tiempo y frecuencia para un día de trabajo en máximo nivel de producción \\ Puesto 1: Desarmado de paleta}

El trabajador por cada paleta que desarma (ciclo) debe flexionar el tronco y el cuello para tomar todos los recipientes que están 
por debajo de la altura de su cadera. Se evidencia que las tres camadas superiores pueden ser tomadas sin inclinación, pero las tres camadas inferiores exigen que el trabajador se incline. Por cada paleta (ciclo) el "trabajador 1" forma 126 pares de envases trasladados que implican 63 flexiones de tronco y cuello con un ángulo de inclinación superior a los 20 grados, 126 rotaciones para el tronco e igual número de flexiones de los miembros superiores (una por cada par de envases tomados). El tiempo que trascurre en una jornada de trabajo a máximo nivel de producción para cada una de estas posiciones son 193, 20 y 267 minutos, respectivamente. Se observa que el trabajador en cuestión, 56\% de la jornada laboral la emplea trasladando los envases hasta la cinta; 40\% del tiempo lo usa para tomar los envases de las camadas ubicadas por debajo de su cadera y sólo 04\% del tiempo lo usa para rotación del tronco. Ver Cuadro № 2.

\section{Cuadro 2.}

Caracterización de las posiciones de trabajo, tiempo y frecuencia observada para el trabajador 1.

\begin{tabular}{|c|c|c|c|c|c|c|c|}
\hline Tarea & Posición & Postura & $\begin{array}{c}\mathrm{N}^{\circ} \\
\text { Repetición }\end{array}$ & $\begin{array}{l}\text { Tiempo } \\
\text { (min.) }\end{array}$ & $\begin{array}{l}\text { Frecuencia } \\
\text { por min. }\end{array}$ & $\%$ & REBA \\
\hline $\begin{array}{l}\text { Recogida } \\
\text { de envases } \\
\text { de paleta }\end{array}$ & $\begin{array}{c}\text { Flexión de tronco } \\
\text { y cuello (recogida } \\
\text { de envases } \\
\text { de la paleta) }\end{array}$ & & 63 & 193 & 0,33 & 40 & $\begin{array}{r}09 \\
\text { Nivel de } \\
\text { riesgo alto } \\
\text { es necesaria }\end{array}$ \\
\hline $\begin{array}{l}\text { Movimiento } \\
\text { de los } \\
\text { envases } \\
\text { hacia } \\
\text { la cinta }\end{array}$ & $\begin{array}{l}\text { Rotación } \\
\text { del tronco }\end{array}$ & & 126 & 20 & 6,30 & 04 & $\begin{array}{r}09 \\
\text { Nivel de } \\
\text { riesgo alto } \\
\text { es necesaria } \\
\text { la actuación } \\
\text { cuanto antes }\end{array}$ \\
\hline $\begin{array}{l}\text { Traslado de } \\
\text { los envases } \\
\text { hasta } \\
\text { la cinta }\end{array}$ & $\begin{array}{l}\text { Flexión de } \\
\text { miembros } \\
\text { superiores }\end{array}$ & & 126 & 267 & 0,47 & 56 & $\begin{array}{r}04 \\
\text { Nivel de } \\
\text { riesgo medio } \\
\text { es necesaria } \\
\text { la actuación }\end{array}$ \\
\hline
\end{tabular}

Carga manejada de un par de envases: rango (9 y 11,5 Kg).

Tiempo total $=8$ horas $=480$ segundos

Fuente: Observación del puesto.

Cuadro 3.

Caracterización de las posiciones de trabajo, tiempo y frecuencia observada para el trabajador 2 .

\begin{tabular}{|c|c|c|c|c|c|c|c|}
\hline Tarea & Posición & Postura & $\begin{array}{c}\mathrm{N}^{\circ} \\
\text { Repetición }\end{array}$ & $\begin{array}{l}\text { Tiempo } \\
\text { (min.) }\end{array}$ & $\begin{array}{l}\text { Frecuencia } \\
\text { por min. }\end{array}$ & $\%$ & REBA \\
\hline $\begin{array}{l}\text { Vigilancia } \\
\text { del proceso }\end{array}$ & Bipedestación & & 63 & 288 & 0,22 & 60 & $\begin{array}{r}02 \\
\text { Nivel de } \\
\text { riesgo bajo } \\
\text { y puede ser } \\
\text { necesaria } \\
\text { la actuación }\end{array}$ \\
\hline $\begin{array}{l}\text { Plastificado } \\
\text { de paquete }\end{array}$ & $\begin{array}{l}\text { Flexiòn de } \\
\text { hombros y } \\
\text { extensión de } \\
\text { miembros } \\
\text { superiores }\end{array}$ & & 63 & 192 & 0,22 & 40 & $\begin{array}{r}05 \\
\text { Nivel de } \\
\text { riesgo medio } \\
\text { es necesaria } \\
\text { la actuación }\end{array}$ \\
\hline
\end{tabular}

Fuente: observación del puesto.
Puesto 2: Plastificación de paquete de envases

El operador de la máquina de plástico (trabajador 2), realiza dos tareas, ellas son la vigilancia del proceso y plastificado de empaques. Él adopta dos posiciones de trabajo, flexión del tronco con inclinación del cuello en un ángulo de $20^{\circ}$ y flexión de los miembros superiores para tomar el plástico con el que luego recubre los envases empaquetados de cuatro en cuatro. El trabajador en un día de trabajo a máxima producción está 288 minutos con flexión de tronco superior a los 20 grados que representa 60\% y 192 minutos está flexionando los miembros superiores, siendo ello el 40\% de su tiempo total de trabajo (ver Cuadro 3).

\section{Puesto 3: Construcción de paletas de producto terminado}

El trabajador del puesto 3, toma los paquetes que son elaborados en el puesto 2, y los coloca sobre la paleta en forma ordenada para formar 6 camadas. Él debe flexionar el tronco a un ángulo mayor de 20 grados y el cuello para colocar todos aquellos paquetes que son depositados por debajo de la altura de su cadera; se observa que las tres camadas inferiores exigen una inclinación del tronco que varía entre 30 y 90 grados; las tres camadas superiores se realizan sin la necesidad de inclinación del trabajador. Por cada paleta (ciclo) el trabajador realiza 63 flexiones de tronco y cuello, 63 rotaciones para el tronco e igual número de flexiones de los miembros superiores (una por cada paquete tomado). El tiempo que trascurre en una jornada de trabajo a máximo nivel de producción para cada una de estas posiciones son 185, 21 y 274 minutos respectivamente. Ver Cuadro 4.

\section{Cuadro 4.}

Caracterización de las posiciones de trabajo, tiempo y frecuencia observada para el trabajador 3.

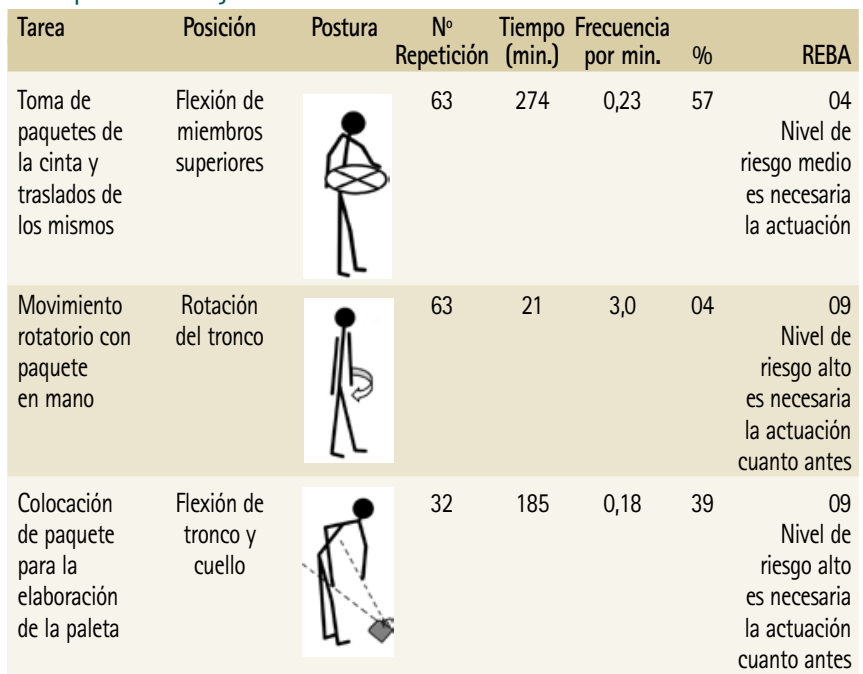

Carga manejada de un par de envases: rango (18 y $23 \mathrm{Kg}$ ).

Tiempo de observación un día de trabajo: 8 horas trabajo y una de descanso. Fuente: observación del puesto.

\section{Evaluación del levantamiento de carga para puestos de Termoencogible aplicando ecuación de NIOSH}

El trabajador del primer puesto, que hace el descargue de la paleta que viene de producción, realiza el levantamiento simultáneo de dos galones; ello representa una manipulación de nueve a once kilos y medio (9-11,5 Kg, según producto en proceso). Una paleta representa 252 galones y exige 126 veces el levantamiento de la carga (dos galones); esto se repite entre 20 y 46 veces al día según 
el nivel de producción. De ello, se tiene que dicho trabajador levanta entre 22680 y $66654 \mathrm{Kg}$. al día. El trabajador del puesto 3 hace el armado de paletas, realiza el levantamiento simultáneo de cuatro galones (un paquete); ello representa una manipulación de 18 a $23 \mathrm{Kg}$., el armado de una paleta representa 252 galones y exige 63 veces el levantamiento de la carga (un paquete); esto se repite entre 20 y 46 veces al día según el nivel de producción. El trabajador levanta entre 22680 y $66654 \mathrm{Kg}$. al día. El análisis de la condición de trabajo para el levantamiento de carga mediante la Ecuación de NIOSH muestra una situación muy peligrosa y alto riesgo de sufrir Trastornos Músculo-Esqueléticos (TME). Ver Cuadro № 05 y 06.

La aplicación de la antropometría muestra condiciones inadecuadas para los trabajadores ya que se presenta un nivel de "no confort"

\section{Cuadro 5.}

Síntesis de la aplicación de la Ecuación de NIOSH para las diferentes tareas en el puesto 1 (manipulación de carga).

$\begin{array}{lcccccc}\text { Altura de la camada } & 27 & 47 & 67 & 87 & 107 & 127 \\ \text { Factor de distancia horizontal (HM) } & 0,694 & 0,694 & 0,694 & 0,694 & 0,694 & 0,694 \\ \text { Factor de altura(VM) } & 0,856 & 0,916 & 0,976 & 0,964 & 0,904 & 0,844 \\ \text { Factor de desplazamiento vertical (DM) } & 0,898 & 0,938 & 1,000 & 1,000 & 1,000 & 0,927 \\ \text { Factor de asimetria (AM) } & 0,808 & 0,808 & 0,808 & 0,808 & 0,808 & 0,808 \\ \text { Factor de frecuencia (FM) } & 0,750 & 0,700 & 0,520 & 0,450 & 0,370 & 0,370 \\ \text { Factor de agarre (CM) } & 0,950 & 0,950 & 0,950 & 0,950 & 0,950 & 0,95 \\ \text { Limite de peso recomendado LPR } & 7,065 & 7,372 & 6,218 & 5,315 & 4,098 & 3,547 \\ \text { FRECUENCIA POR MINUTOS } & 6 & 7 & 9 & 10 & 12 & 12 \\ \text { İndice de levantamiento } & 1,62 & 1,55 & 0,54 & 2,16 & 2,8 & 3,24\end{array}$

Carga manejada $11,5 \mathrm{Kg}$. LC 13,8.

\section{Cuadro 6.}

Síntesis de la aplicación de la Ecuación de NIOSH para las diferentes tareas en el puesto 3 (manipulación de carga).

$\begin{array}{lcccccc}\text { Altura de la camada } & 127 & 107 & 87 & 67 & 47 & 27 \\ \text { Factor de distancia horizontal } & 0,625 & 0,625 & 0,625 & 0,625 & 0,625 & 0,625 \\ \text { Factor de altura } & 0,844 & 0,904 & 0,964 & 0,976 & 0,916 & 0,856 \\ \text { Factor de desplazamiento vertical } & 0,927 & 1,000 & 1,000 & 1,000 & 0,938 & 0,898 \\ \text { Factor de asimetria } & 0,856 & 0,856 & 0,856 & 0,856 & 0,856 & 0,856 \\ \text { Factor de frecuencia } & 0,800 & 0,800 & 0,700 & 0,700 & 0,520 & 0,520 \\ \text { Factor de agarre } & 0,950 & 0,950 & 0,950 & 0,950 & 0,950 & 0,950 \\ \text { LPR } & 7,317 & 8,454 & 7,888 & 7,986 & 5,223 & 4,673 \\ \text { FRECUENCIA POR MINUTOS } & 5 & 5 & 7 & 7 & 9 & 9 \\ \text { İndice de levantamiento } & 3,14 & 2,72 & 2,91 & 2,88 & 4,40 & 4,92\end{array}$

Carga manejada $23 \mathrm{Kg}$. $\mathrm{LC} \geq 60$.

\section{Cuadro 7.}

Determinación del confort considerando simultáneamente el tipo de trabajo y la altura (exigencia alta, ligera-moderada) para los trabajadores del área Termoencogible.

\begin{tabular}{|c|c|c|c|c|c|c|c|c|c|c|c|c|c|c|c|}
\hline \multicolumn{7}{|c|}{ CARACTERISTICAS DE LOS TRABAJADORES EVALUADOS } & \multicolumn{8}{|c|}{ ALTURA DE TRABAJO PARA LAS TAREAS (M) } & \multirow{3}{*}{$\begin{array}{l}\text { Criterios Análisis } \\
\text { \% confort } \\
\text { Ideal }\end{array}$} \\
\hline \multirow[t]{2}{*}{$\mathrm{N}^{\circ}$} & \multirow{2}{*}{\multicolumn{2}{|c|}{$\begin{array}{l}\text { Variables personales } \\
\text { Talla }(\mathrm{m}) \text { Peso } \mathrm{Kg}\end{array}$}} & \multirow[t]{2}{*}{ IMC } & \multirow{2}{*}{\multicolumn{3}{|c|}{$\begin{array}{l}\text { Dimensiones corporales } \\
\text { Drillis \& Contini, (1966) (metros) } \\
\text { Nudillo de dedos Codos Hombros } \\
\text { (Manos) }\end{array}$}} & \multicolumn{6}{|c|}{$\begin{array}{c}\text { Puestos } 1 \text { y } 3 \\
\text { Tarea de Manipulación de camadas }\end{array}$} & \multicolumn{2}{|c|}{$\begin{array}{c}\text { Puesto № } 2 \\
\text { Plastificado de paquetes }\end{array}$} & \\
\hline & & & & & & & & & 0,67 & 0,87 & 1,07 & 1,27 & & & \\
\hline 2 & 1,75 & 71 & 23 & 0,66 & 1,10 & 1,43 & - & - & $+/-$ & + & $+/-$ & $+1-$ & - & + & 25 \\
\hline 3 & 1,78 & 78 & 25 & 0,67 & 1,12 & 1,46 & - & - & $+1-$ & + & $+1-$ & $+1-$ & - & + & 25 \\
\hline 4 & 1,80 & 95 & 29 & 0,68 & 1,13 & 1,47 & - & - & $+1-$ & + & $+1-$ & $+1-$ & - & + & 25 \\
\hline
\end{tabular}

- la altura de trabajo carece de confort. + La altura de trabajo proporciona confort. + +-La altura de trabajo a mediano confort

Rango de confort ideal: codos y nudillos de las manos. Rango de mediano confort: codos -hombros y manos-rodillas. Rango de no confort: por debajo de rodillas y por encima de hombros.

Fuente: elaboración propia. preocupante, esto es de 37,5\% y mediano confort de 37,5\%, lo que indica que la mayoría de las posiciones de trabajo adolece de una inadecuación de las dimensiones del puesto con las características antropométricas de los trabajadores (ver Cuadro 07) lo que a su vez obliga a los trabajadores a realizar tareas con adopción de posturas incómodas, peligrosas que exige una actuación ergonómica (ver Cuadro № 08)

\section{Cuadro 8.}

Recomendaciones para mejorar las condiciones de trabajo del área de Termoencogible.

\begin{tabular}{|c|c|c|c|}
\hline $\begin{array}{l}\text { ¿Qué hacer } \\
\text { en concreto? }\end{array}$ & $\begin{array}{l}\text { Consideraciones que } \\
\text { justifican la acción }\end{array}$ & Responsables & $\begin{array}{l}\text { Otros Aspectos } \\
\text { a estudiar }\end{array}$ \\
\hline $\begin{array}{l}\text { Incorporar ayuda mecánica } \\
\text { para armado y desarmado } \\
\text { de paletas o, en su defecto, } \\
\text { modificar los niveles de } \\
\text { altura para los puestos de } \\
\text { trabajo que exigen manipu- } \\
\text { lación de carga incorporan- } \\
\text { do una grúa o andamio, } \\
\text { mesa giratoria y basculante } \\
\text { que permita subir y bajar la } \\
\text { altura de las paletas depen- } \\
\text { diendo del peso soportado } \\
\text { según las necesidades de } \\
\text { los trabajadores. } \\
\text { (Puesto } 1 \text { y 3). }\end{array}$ & $\begin{array}{l}\text { Control de la fuente de riesgo } \\
\text { para TME. } \\
\text { Deficiencias en cumplimento de } \\
\text { las leyes. } \\
\text { Déparis establece condición de peligro } \\
\text { por adecuación del trabajo, ritmo de } \\
\text { trabajo, manipulación de carga y fac- } \\
\text { tores psicosociales. NIOSH indica que } \\
\text { la carga de trabajo supera la permiti- } \\
\text { da, por tanto, hay riesgo para TME. } \\
\text { REBA indica que existe riesgo en las } \\
\text { posturas de trabajo. } \\
\text { Existen debilidades de confort, } \\
37,5 \% \text { de las alturas de trabajo } \\
\text { están adecuadas. }\end{array}$ & $\begin{array}{l}\text { Departamento de } \\
\text { Salud y Seguridad } \\
\text { en el Trabajo. } \\
\text { Gerencia de la } \\
\text { empresa. }\end{array}$ & $\begin{array}{l}\text { Entrenamiento } \\
\text { de los trabajado- } \\
\text { res para el uso } \\
\text { de la máquina } \\
\text { incorporada. }\end{array}$ \\
\hline $\begin{array}{l}\text { Disminuir el ritmo de traba- } \\
\text { jo regulando la velocidad de } \\
\text { la cinta transportadora e } \\
\text { incorporando personal } \\
\text { para cumplir con las } \\
\text { exigencias de producción. } \\
\text { Tomar en vez de dos } \\
\text { galones solo } 1 .\end{array}$ & $\begin{array}{l}\text { El ritmo de trabajo los define el } \\
\text { movimiento de la cinta y exige a } \\
\text { los trabajadores rapidez y le da una } \\
\text { referencia al supervisor para } \\
\text { apurarlos. }\end{array}$ & $\begin{array}{l}\text { Recursos } \\
\text { Humanos y } \\
\text { Gerencia de } \\
\text { Producción } \\
\text { y trabajadores } \\
\text { del área. }\end{array}$ & $\begin{array}{l}\text { Velocidad de } \\
\text { referencia de } \\
\text { la cinta } \\
\text { transportadora. }\end{array}$ \\
\hline $\begin{array}{l}\text { Modificar el nivel de refe- } \\
\text { rencia para tomar el plástico } \\
\text { de envoltura del puesto } 2 \text {. }\end{array}$ & $\begin{array}{l}\text { La elevación de miembros superiores } \\
\text { de forma repetitiva que sale del } \\
\text { nivel de confort. }\end{array}$ & $\begin{array}{l}\text { Gerencia General } \\
\text { y trabajadores del } \\
\text { área. }\end{array}$ & \\
\hline $\begin{array}{l}\text { Diseñar y aplicar un plan } \\
\text { de prevención de TME } \\
\text { incorporando pausas de } \\
\text { trabajo, rotación del perso- } \\
\text { nal y educación para la } \\
\text { adopción de posturas. }\end{array}$ & $\begin{array}{l}\text { La educación y la prevención son } \\
\text { las herramientas para prevenir los } \\
\text { accidentes y las enfermedades } \\
\text { ocupacionales. }\end{array}$ & $\begin{array}{l}\text { Médico especia- } \\
\text { lista en salud } \\
\text { ocupacional. } \\
\text { Recursos } \\
\text { Humanos. }\end{array}$ & $\begin{array}{l}\text { Necesidades parti- } \\
\text { culares de los tra- } \\
\text { bajadores. }\end{array}$ \\
\hline $\begin{array}{l}\text { Considerar el rediseño del } \\
\text { puesto } 2 \text { para propiciar el } \\
\text { trabajo en posición de } \\
\text { sedestación o en forma } \\
\text { mixta para evitar posturas } \\
\text { prolongadas. }\end{array}$ & $\begin{array}{l}\text { Falta de confort para los miembros } \\
\text { superiores. }\end{array}$ & $\begin{array}{l}\text { Departamento de } \\
\text { Salud y Seguridad } \\
\text { en el Trabajo y } \\
\text { Recursos } \\
\text { Humanos. }\end{array}$ & \\
\hline $\begin{array}{l}\text { Instalar un sistema de } \\
\text { ventilación para la zona de } \\
\text { producción evitando la } \\
\text { acumulación de químicos } \\
\text { en las áreas de trabajo. }\end{array}$ & $\begin{array}{l}\text { La exposición a riesgos quimicos } \\
\text { puede afectar la salud de los trabaja- } \\
\text { dores del área de Termoencogible y } \\
\text { en mayor medida a los de la zona } \\
\text { donde se manipulan tales sustancias. }\end{array}$ & $\begin{array}{l}\text { Departamento } \\
\text { de salud y seguri- } \\
\text { dad en el trabajo. } \\
\text { Gerencia General. }\end{array}$ & $\begin{array}{l}\text { Concentración } \\
\text { de químicos en } \\
\text { las áreas de } \\
\text { trabajo y su } \\
\text { toxicidad. }\end{array}$ \\
\hline
\end{tabular}

Fuente: entrevista a los trabajadores. 


\section{DISCUSIÓN DE RESULTADOS}

Los trabajadores refieren debilidades en la adecuación del trabajo, el mismo es repetitivo y representa peligros físicos y psicosociales, implica la manipulación de carga (9 a $23 \mathrm{Kg}$ ) en situación de "contrarreloj" y supervisión estricta. Respecto del diagnóstico de las condiciones de trabajo son Insatisfactorias y Peligrosas, que incluyen una Mediana para mejorar. El trabajador del Primer puesto (Puesto 1), presenta un riesgo alto para Trastornos Musculoesqueléticos en la tarea de recogida de envases, se hace necesaria la actuación cuanto antes; y, en el traslado de los envases, se determinó que presenta un riesgo medio; por ello, la actuación es necesaria. El trabajador del puesto dos (Puesto 2), durante la vigilancia del proceso se expone a un riesgo bajo y una actuación ergonómica pondría ser necesaria. El plastificado de paquete tiene un nivel de riesgo medio que define una actuación ergonómica necesaria. El trabajador del tercer puesto (Puesto 3) presenta una situación similar al Puesto 1. Los trabajadores en el primer y tercer puesto realizan tareas con un nivel de riesgo alto para los TME. Al comparar la situación de puesto 3 y 1, la carga manejada por el trabajador 3 es mayor (se duplica), agravando el riesgo; ambos se exponen a la adopción de posturas incómodas, manipulación de carga y alto grado de repetitividad (ver cuadros $1,2,3,4)$.

Los puestos de trabajo 1 y 3 presentan tareas donde existe un incremento acusado del riesgo (Índice de levantamiento $>3$ ). Este tipo de tarea es inaceptable desde el punto de vista ergonómico y debe ser modificada (ver Cuadro № 05 y 06). El trabajador del área de empaquetado (Puesto 2) parece tener mejores condiciones de trabajo en comparación con los puestos vinculados con el descargue y armado de paletas, no exigen la manipulación de carga; sin embargo, la vigilancia del proceso y el plastificado es un trabajo realizado en estado de bipedestación prolongada, con excesiva supervisión, en contrarreloj y que no permite el descanso. Es un estado de riesgo bajo que puede exigir una actuación. Resulta interesante considerar que las zonas de dolor y fatiga al final de la jornada indican que la espalda baja, los hombros, la espalda alta y el cuello son las regiones del cuerpo que se perciben afectadas luego de la jornada. Al respecto, la prevención de los trastornos lumbares relacionados con el trabajo exige: diseño ergonómico del trabajo, educación y formación, $\mathrm{y}$ selección del trabajador. ${ }^{9,10}$

Las condiciones de trabajo en el área determinan trastornos de tipo músculo-esqueléticos. Al respecto, Montiel, Romero, Lubo y otros ${ }^{11}$, refieren que al estar presente la manipulación de carga, el trabajo repetitivo en contrarreloj, un ambiente psicosocial inadecuado y peligroso y la adopción de posturas incómodas que, a su vez, interactúan de forma simultánea para afectar la salud de los trabajadores y generar TMT. Por ello, se requiere una modificación urgente de los puestos de trabajo que permita la adecuación del puesto a las dimensiones de los trabajadores para: a) evitar daños a la salud y las pérdidas económicas vinculadas por demandas por enfermedades ocupacionales. Los determinantes presentes en el puesto de trabajo que podrian explicar las lesiones musculoesqueléticas son los problemas de organización del trabajo referidos a la supervisión estricta, carga de trabajo excesiva, déficit de personal, siendo el diseño del puesto inadecuado, se hace necesario evitar la manipulación de cargas fuera de la zona neutral del cuerpo.

Trabajo ganador del Primer Lugar del Congreso Internacional de Prevención de Riesgos Laborales ORP 2012

\section{REFERENCIAS}

1. Esser J, Rojas C. Subjetividad, trabajo y salud mental. Salud de los Trabajadores 2006; 14(2):81-85.

2. Nataren J, Noriega M. Los trastornos músculo-esqueléticos y la fatiga como indicadores de deficiencias ergonómicas y en la organización del trabajo. Salud de los Trabajadores. 2004;12(2):27-41.

3. Yanes $L$. La salud de los trabajadores en el marco del proceso político venezolano: Impacto de la reforma de la ley orgánica de prevención, condiciones y medio ambiente de trabajo. Salud de los Trabajadores. 2009;16(2):103-113.

4. Escalona $E_{1}$ Young $M$, González $R$, Chatigny $C$, Seifert A. La ergonomía como herramienta para trabajadoras y trabajadores. Valencia-Venezuela: Universidad de Carabobo; 2002.

5. Messing K, Vogel L, Parra M, Grönkvis L, Lagerlöf E. El trabajo de las mujeres: comprender para transformar. Madrid: Instituto de la Mujer-Ministerio de Trabajo y Asuntos Sociales; 2002.

6. Malchaire J. Strategie Sobane et Guide de Depistage Deparis [en línea]. Paris:CEEUnité Hygiéne et Physiologie du Travail; 2007 [citado 3 may 2009]. Disponible en: http://www.deparisnet.be/sobane/fr/brochure_sobane_2007_fr.pdf

7. Diego-Más JA, Asencio M. Método REBA (Rapid Entire Body Assessment). Ergonautas.com [en línea]. Valencia: Universidad Politécnica de Valencia; 2006 [citado 7 mar 2009]. Disponible en: http://www.ergonautas.upv.es/metodos/ reba/reba-ayuda.php

8. Castillo V, Escalona E. Cuando el trabajo en oficinas se percibe pesado: Casos en una universidad venezolana. Salud de los Trabajadores. 2009;2(17):107-120.

9. Kovacs FM, Gestoso M, Mufraggi N, Gil del Real MT, Santos S, Martín M. Programa de Mejora de la Salud Laboral y Reducción del Absentismo por lumbalgia inespecifica. Medicina del Trabajo (AEEMT). 2000;9(5):211-20 .

10. Guzmán A, Borgas L, Muñoz B. Determinación de Factores de Riesgo ocupacional generadores de Lumbalgia mecánica en cosechadores de madera. Revista F.C.S. [en linea] 2007; 9(1):15-20 [citado 1 dic 2009]. Disponible en: http://www.facultadsalud.unicauca.edu.co/fcs/2007/marzo/revistamarzo.html.

11. Montiel M, Romero J, Lubo A, Quevedo L, Rojas L, Chacin B, Sanabria C. Valoración de la carga postural y riesgo musculoesqueléticos en trabajadores de una empresa metalmecánica. Salud de los Trabajadores. 2006;14(1):61-69.

\section{Referencia Complementaria:}

1. Drillis R, Contini R. Body segment parameters. New York: School of Engineering and Science; 1966. 24 p. Technical Report; PB174945, 